\title{
Optimizing Power-Based Indoor Tracking System for Wireless Sensor Networks using ZigBee
}

\author{
Ahmad H. Mahafzah \\ Department of Computer Science \\ Faculty of Information technology \\ Middle East University Amman, 11831 Jordan
}

\author{
Hesham Abusaimeh \\ Associate Professor of Computer Science \\ Faculty of Information Technology \\ Middle East University Amman, 11831 Jordan
}

\begin{abstract}
Evolution of wireless and digital communication gives birth to the smaller but powerful battery-equipped devices which are easy to maintain and perform the desired tasks. ZigBee is a Wireless Personal Area Network (WPAN) used for home or indoor automation, collecting data for medical research by using the low power digital radios to handle the low data rate. In ZigBee network, sensor nodes are heterogeneously deployed and continuously moving. To detect and tracking of those sensor nodes are challenging in terms of accuracy, calculation time and energy consumption. In this paper, proposed system uses the Received Signal Strength Indication (RSSI) protocol for localization, trilateration for fetching the exact coordinates of sensor nodes and these protocols helps to overcome the problem which eventually led to prolonged sensor network, accurate localization.
\end{abstract}

Keywords-Indoor tracking; ZigBee; wireless personal area network; localization; trilateration

\section{INTRODUCTION}

WSN consists of multiple heterogeneous and homogeneous sensor nodes [1]. The basic difference between these two are consists in their basic feature i.e. initial power, processing capability, signal strength etc. These nodes are used to gather the data from the surrounding. Usually, the nodes in range share data with each other to communicate better and transmit the gathered data to Sink Node (SN). Sink node is used as the intermediary or gateway node between the WSN and the researchers. As suggested by [2], sensor nodes store the data processing and communication protocols to process them when required.

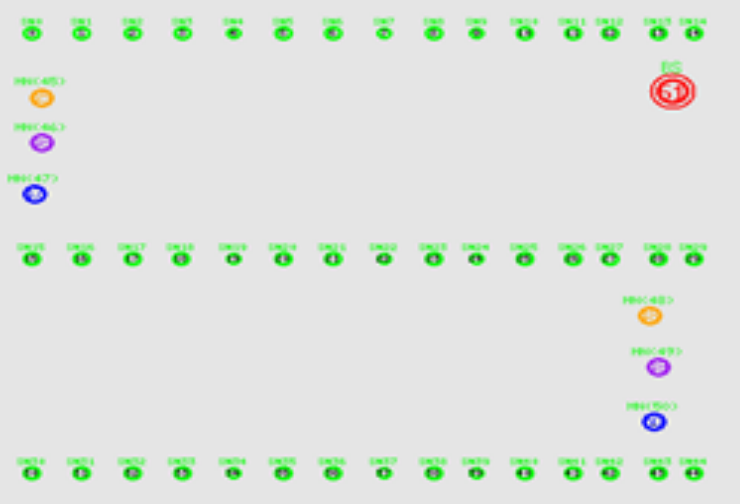

Fig. 1. ZigBee Wireless Sensor Network.
Sensor nodes are randomly deployed in a WSN as shown in figure 1. Each sensor node has a responsibility to collect data from sensor field, share it with other and finally route the collected data to the base station or sink node. As having a mesh network, collected data will be routed back to end user by a multi-hop infrastructure. And in the end, it is the responsibility of sink node to send the data to the user.

Each sensor node consists of battery, sensor unit, memory unit, transceiver (omniAntenna) and processor unit. These parameters have their own unique function to perform in the network. Battery-equipped sensor nodes are used to provide the power; sensor unit uses the omniAntenaa for transceiver for sensing the environment and collect the desired data packets. After processing the data, memory unit will help them to keep the data safe. As sensor nodes has small size memory unit, they cannot save it for long and sensor nodes have to override the memory segments which may cause data loss. So, nodes will transmit the data packets to sink node very frequently to save the data and avoid the anomalies [3].

The WSN is available to provide many services like indoor tracking mobile object through ZigBee network standard. ZigBee network provides remarkable communication protocols using low range and low-power digital radios based on standard IEEE 802.15.4 for Wireless Personal Area Networks. It is originally designed to provide short-range communication services that provides lower data rate. ZigBee is developed on the network and application layer in IEEE 802.15.4 standard. Topologies is considered as an acceptable three main of configuration, they are tree, star and mesh topologies. It is built on top of IEEE 802.15.4 standard, which defines the characteristics of the physical and Medium Access Control (MAC) layer on protocol stack for WPAN [4].

Node localization is defined as determining the position of a sensor node in the concerned network area with respect to origin. Localization techniques for WSN consist of the algorithms that estimate the locations of sensors with initially unknown position information normally using available information about the absolute positions of a few other sensors and position measurements. Sensors with known location information are called beacons or anchors. The anchors define the local coordinate system to which all other sensors are referred. The coordinates of the sensors with unknown location information also called blind or non-anchor nodes, it is estimated by various sensor network localization techniques. The most popular method in distance estimation 
for wireless systems is the Received Signal Strength Indicator (RSSI) technique, which is based on the physical fact of wireless communication that theoretically, the signal strength is inversely proportional to the squared distance between the transmitter and receiver [5].

\section{RELATED WORK}

Chen et al. [6] presented a survey with reference to RSSI solutions on indoor localization and proposed a Closer Tracking Algorithm (CTA) to locate a mobile user in the house. The proposed CTA was implemented by using ZigBee CC2431 modules. The proposed CTA shows at least $85 \%$ precision when the distance is less than one meter.

Larranaga et al. [7] discussed different environmental factors that affect the RSSI values measured to calculate the position of the device. Proposed system consists of two main phases: calibration and localization. The use of a central processing server allows the implementation of complex algorithms, while the ZigBee network allows collecting signal level values and at the same time, it is used to provide data to the central server for localization computation. To locate blind nod the system performs the calibration, so that changes in the environment are taken into account in the localization phase, and thus making the system more robust and accurate.

Chu et al. [8] discussed the two novel techniques to improve tracking in indoor system using ZigBee by improving positioning accuracy. Authors combined the Neighbour Area Majority Vote Priority Correction and Environment Parameter Correction. The experiment results demonstrate that proposed methods can largely increase accuracy of ZigBee positioning and provide useful personnel tracking technology.

Chandane et al. [9] analysed performance of IEEE 802.15.4 using Qualnet 4.5. Author designed star topology, multi-hop peer to peer network. MANET routing protocols such as AODV, DSR and DYMO are used for analysis of Quality of Service (QoS) parameters like throughput, packet delivery ratio, average end-to-end delay, jitter, total energy consumption, and network scalability as the performance metrics. Results show that AODV outperforms other two in star topology whereas DSR has slightly upper hand in multihop topology for varying traffic loads and in beacon-enabled mode.

Obaid et al. [10] briefly explained an overview of ZigBee technology and its application in wireless home automation systems. The performances of the ZigBee based systems have also been compared with those of other competing technologies-based systems. In addition, some future opportunities and challenges of the ZigBee based systems have been listed Oracevic \&Ozdemir (2014) targeted different target tracking methods via focusing on security. Author explained the important protocols in each category and described which security properties they provide. Paper presented that most of the research in target tracking focused on the accuracy or energy efficiency and there is limited amount of work that considers security. Author presented a table that summarizes the state of the art in the target tracking area.
Vancin \& Erdem [11] in this study utilize the IEEE 802.15.4/ZigBee, which has advantages than other types of technology with respect to parameters such as; use of the battery in addition to low consumption of power. In this study, OPNET simulator is used to achieve the required results. The behaviour of mobile node and network fixed has been compared based on the quality of the end-to-end delay parameters and traffic received through the destination.

Shinde et al. [12] created a study in order to develop a low cost, indoor location positioning system that can be utilized to find the indoor moving position of the object. This paper portrays a novel low-cost system for two diminution (2D) locations monitoring utilizing indoor ZigBee Technology through location fingerprinting technique to estimate position. Use of Mesh networking of ZigBee assesses in making the network more scalable and also in increasing the coverage area of the system.

TABLE I. SimULATION PARAMETERS

\begin{tabular}{|l|l|l|}
\hline Serial No. & \multicolumn{1}{|c|}{ Parameter Name } & \multicolumn{1}{c|}{ Parameter Value } \\
\hline 1. & Area of flat grid & $400 \mathrm{~m} * 400 \mathrm{~m}$ \\
\hline 2. & No. of sensor nodes & 45 \\
\hline 3. & No. of mobile nodes & 6 \\
\hline 4. & No. of Base Station & 1 \\
\hline 5. & MAC Layer Protocol & MAC/802.15.4 \\
\hline 6. & Simulation time & $100 \mathrm{~s}$ \\
\hline 7. & Antenna type & OmniAntenna \\
\hline 8. & Radio model & TowRayGround \\
\hline 9. & Transmission range & $30 \mathrm{~m}$ \\
\hline 10. & Routing protocol & AODV \\
\hline 11. & energyModel & $100 \mathrm{Joules}$ \\
\hline 12. & T power & $1.0 \mathrm{w}$ \\
\hline 13. & R power & $1.0 \mathrm{w}$ \\
\hline 14. & DataSize & $1000 \mathrm{Byte}$ \\
\hline 15. & DataRate & $5 \mathrm{~K}$ bits/s \\
\hline
\end{tabular}

\section{EXPERIMENTAL DESIGN}

Existing system lacks the accuracy while fetching the coordinates of sensor nodes and very prone to choke the battery very fast. Proposed model removes these errors and drawbacks by the combination of trilateration, localization and energy consumption. Trilateration is used to get the locations of different sensor nodes and store the coordinates into a trace file. This will help the sensor network to know the initial location of nodes. After initiating the simulation, mobile nodes 45to 47 will start moving randomly towards the sink and mobile nodes 48 to 50 away from the sink node. This will provide robust scenario of mobility in both directions. Just 
after initiating the simulation to transfer data packets, a hello packet is broadcasts to every node in the network and simultaneously RSSI procedure starts working. RSSI computes the distance between mobile node and sensor nodes and stores them into a trace file at every 0.5 seconds time interval. The rest of simulation parameters are defined such as mentioned in the following Table 1.

\section{ALGORITHM}

The proposed model uses the localization and distance techniques to get the exact location and distance among them and storing the location coordinates and distance into a trace file.

Figure 2 describes the working of proposed model. These two tables are used to send the data from mobile nodes to sink node.

To transfer the data uninterruptedly, they use the nearby sensor nodes as intermediates and send it. Now sink node has the sensed data of network and the location of nodes that is stored in different tables.

\section{PROPOSED ALGORITHM}

1. Node N starts up.

2. Start mobility of Mobile nodes (from 45 to 50).

$M N=\left\{\right.$ node_N $X_{\text {coordinate }} Y_{\text {coordinate }}$ Speed $\}$

3. Get location using trilateration

4. Calculate Distance between sensor node and Mobile node

$d=\sqrt{(\mathrm{X} 2-\mathrm{X} 1)^{2}+(\mathrm{Y} 2-\mathrm{Y} 1)^{2}}$

Where $X 1$ and $X 2$ is $X$-axis of sensor nodes and $Y 1$ and $Y 2$ are $\mathrm{Y}$-axis of sensor nodes

5. Build distance table

6. Calculate remaining energy (RemEng) of mobile node Pt $=$ PtInit $\times \frac{\text { RemEng }}{\text { InitEng }}$

$$
\text { RemEng } \quad=P t \times \operatorname{GTX} \times \operatorname{GRX}\left(\frac{\lambda}{4 \pi d}\right)^{2}
$$

Where $P t$ is transmission power; PrInit is the initial reception power, RemEng is the remaining energy of the node, and InitEng is the initial full energy of the node

7. Build Residual Energy table.

8. Compute the Received Signal Strength Indicator (RSSI). $\quad R S S I=10 \times L O G \frac{\text { RemEng }}{\text { PRef }},[R S S=$ $d B m]$
Where RemEng is remaining energy, PRef is reference power.

9. Update distance table

10. Start data transmission between nodes.

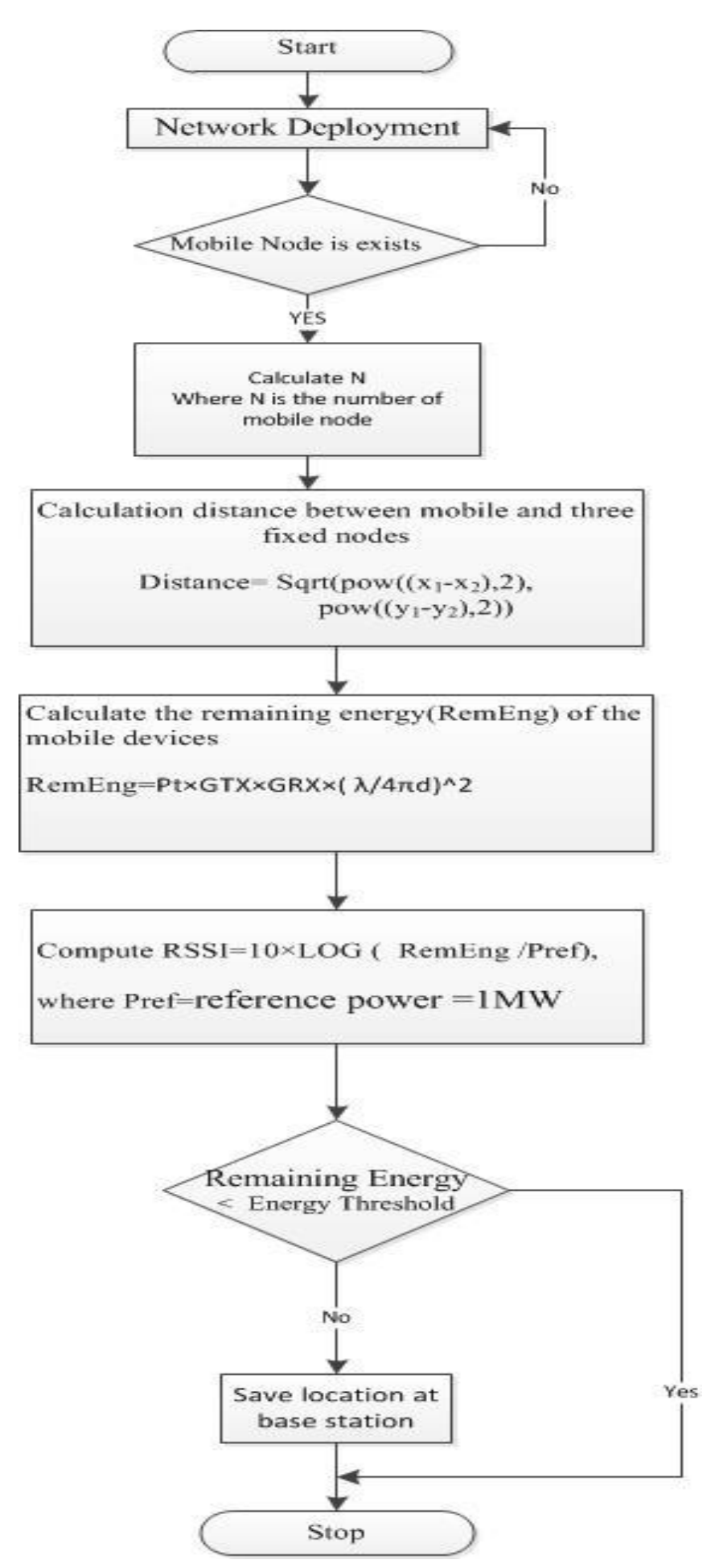

Fig. 2. Flowchart of Proposed Model. 


\section{RESUlts}

The proposed model has been implemented using the Network Simulator version 2.35 (NS2). There are 52 sensor nodes deployed randomly in the wireless network where nodes ranging from 0 to 44 are sensor nodes, nodes ranging from 46 to 50 are mobile nodes and node 51 is programmed as sink node. Where sensor nodes are static in nature and mobility is defined randomly. The sensing and transceiver range is fixed to 30 meter as the property of ZigBee.

Table 2 describes the average results and performance evaluation of existing and proposed systems. This clearly shows that the proposed system is better in every aspect and provides more reliable sensor network.

TABLE II. PERFormanCE EVALUATION OF PROPOSED AND EXISTING SYSTEM

\begin{tabular}{|l|l|l|l|}
\hline Parameters & $\begin{array}{l}\text { Proposed } \\
\text { system } \\
\text { (average) }\end{array}$ & $\begin{array}{l}\text { Existing } \\
\text { system } \\
\text { (average) }\end{array}$ & $\begin{array}{l}\text { Improvement } \\
\text { (approx.) }\end{array}$ \\
\hline Tracking Error & 0.73 & 0.79 & $8 \%$ \\
\hline $\begin{array}{l}\text { Throughput(bit/second } \\
\text { (bps)) }\end{array}$ & 91095 & 63020 & $45 \%$ \\
\hline Delay(millisecond(ms)) & 2 & 3.1 & $36 \%$ \\
\hline PDR(bit/second (bps)) & 2107.1 & 1400 & $73 \%$ \\
\hline $\begin{array}{l}\text { Residual Energy } \\
\text { joules) }\end{array}$ & 74.7 & 69.9 & $7 \%$ \\
\hline
\end{tabular}

A. Throughput

Throughput can be computed in Gbps, Mbps and Kbps. This shows the successfully transferred number of packets from source to destination.

Figure 3 shows that the packets throughput during the simulation is very high as compared to existing system because of the localizing the sensor nodes. This allows them to know the exact location of each node and reduce the time and connection packets to delivery.

\section{Throughput}

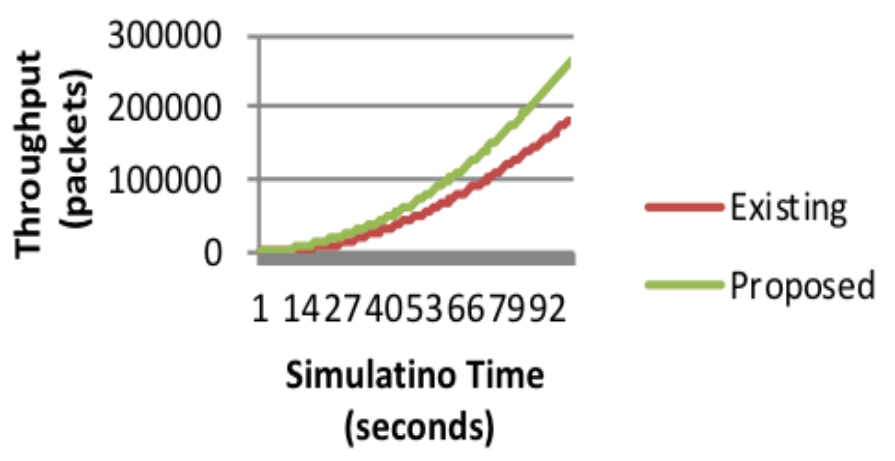

Fig. 3. Throughput of Proposed System.

\section{Delay}

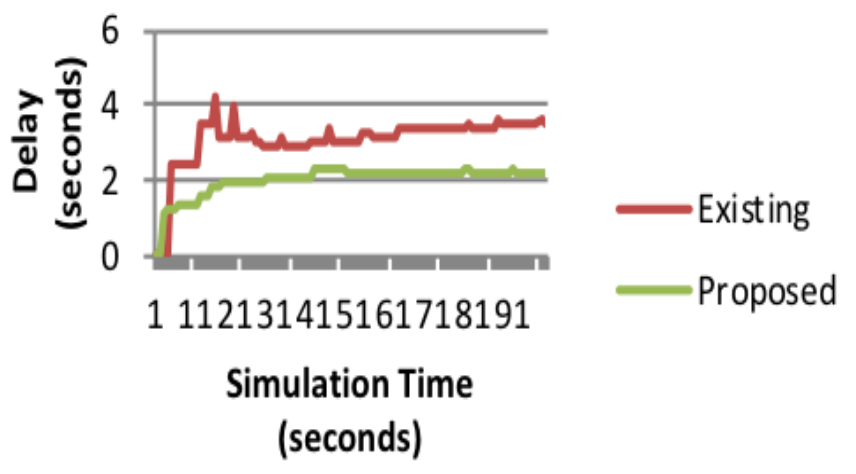

Fig. 4. Delay of Existing Proposed System.

\section{B. Delay}

Delay shows the delay of packets from source to destination. This can be caused because of queuing of packets, route discovery, low transmission rate etc. only successful data packets are considered during simulation.

Figure 4 shows that the delay packets is reduced by the proposed model and controlled after a sudden hike into it. The delay can be caused by multiple factors like sending sensing signals to gather information about neighbours, storing them into a table, parsing the table to get the nearest node to transfer data bytes.

\section{Packet Delivery Ratio}

The packet delivery ratio can be defined as the ratio of packets successfully received to the total sent. Figure 5 shows the efficiency of the network.

Proposed system has high Packet delivery ratio than existing because of their applied algorithms that proposed system don't have to create connection every time they want to send packets. There are distance and connection trace files available eases the path formation procedure.

\section{Packet Delivery Ratio}

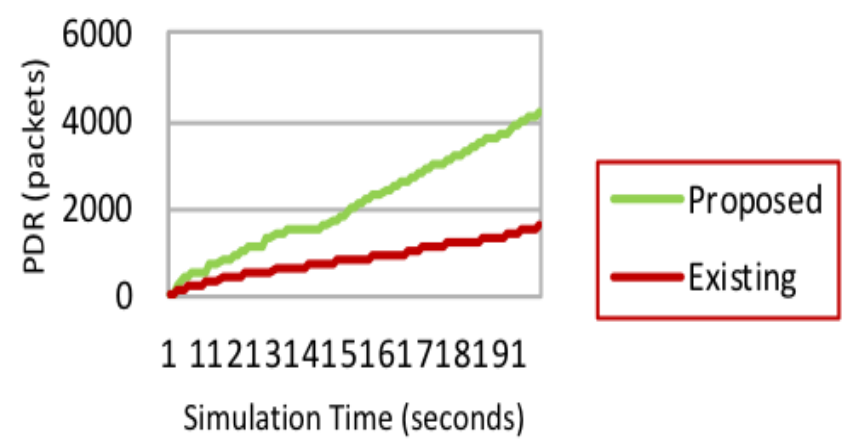

Fig. 5. Packet Delivery Ratio of Proposed System. 


\section{Energy Consumption}

Residual energy is the remaining energy of any sensor node. Figure 6 will show that the lifetime of node or any network.

The topography patter of both system are different nature and existing system used less number of sensor nodes and the deployment of nodes are also half the size of proposed system. Due to this topography and nodes deployment of both systems, we can deduce that proposed system can survive twice the existing system.

\section{Residual Energy of Sink Node}

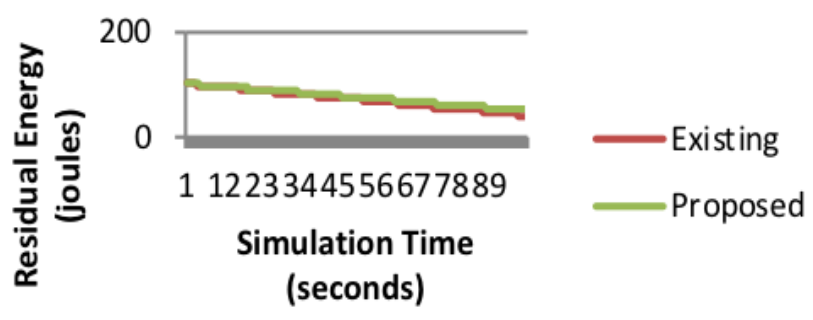

Fig. 6. Residual Energy of Sink Node.

\section{E. Accuracy}

Accuracy of finding the location it is measured by taking the averaged Euclidean distances between the real coordinates and estimated coordinates. It presents the uncertainty of having the correct target's location.

\section{Avarage Tracking Error}

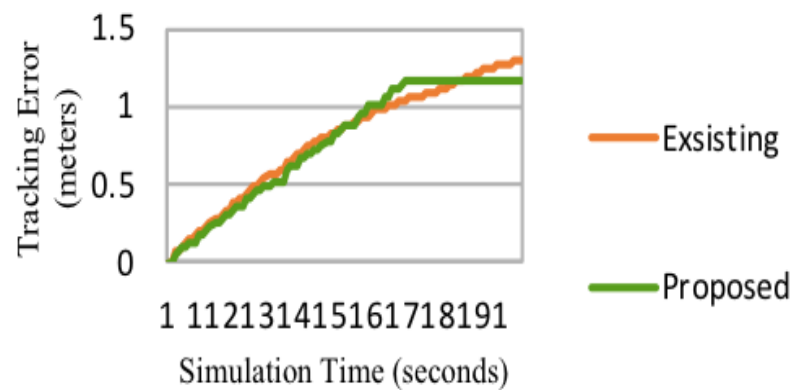

Fig. 7. Average Accuracy of all Mobile Nodes.

The accuracy of finding the distance is reduced by considering the trilateration algorithm. Figure 7 shows the accuracy of proposed system increases gradually because of the proposed algorithm.

\section{CONCLUSION}

This thesis presents Indoor tracking using ZigBee Wireless Sensor Network. The proposed algorithm is designed after considering all the loopholes which may affect the environment. Heterogeneous sensor nodes are deployed in the
ZigBee wireless sensor network to measure the effectiveness and efficiency of the proposed system. The proposed system can divide the implantation of algorithm into mainly three parts i.e. ensuring the availability of sensor nodes while connection and path formation by considering the residual energy of sensor node and store the energy into a trace file; fetching the locations of sensor nodes, mobile nodes and sink node using trilateration technique and store the location into a trace file and in the end, using Received Signal Strength Indicator (RSSI) technique determine the distance between each and every sensor node, mobile node and sink node and store the distance into a trace file. This process iterates itself after every 0.5 seconds. The trace tables of energy and distance are updated and provide the exact figures throughout the simulation. The proposed model is designed to eradicate the drawbacks of the existing system by merging the numerous algorithms together to develop a new and improved solution. The proposed model improved the indoor tracking systems lifetime using ZigBee wireless sensor network. This will also help to prolong the existence of network and work to sense and transmit data for a long time. The proposed model works on the principle of collecting information first rather than sending it. The calculation of numerous parameters like localization or distance and building the centralized trace table for the sensor nodes to provide the identical information to each node helps the proposed system to prolong the lifetime of the sensor network and more importantly increases the accuracy of the system. To get the assurance of working efficiency, effectiveness and accuracy of the proposed model, it is examined for various parameters like throughput, delay, residual energy and accuracy. The combination of examined collected data, trilateration and RSSI for tracking the indoor scenarios makes it more adaptable and effective than others.

A performance result of this method has been measured and compared with the original RSSI method using NS-2 simulator. All the achieved simulation results have shown better performance for the proposed methods as compared to the original method. The Tracking Error improved by $8 \%$ and power consumption improved by $7 \%$. Furthermore, the new network performance measurements have obtained a good value compared with the other measures in term of the network performance measurements such as packet delivery ratio (PDR), delay and throughput improved by $73 \%, 36 \%$ and $45 \%$, respectively.

\section{ACKNOWLEDGMENT}

The authors are grateful to the Middle East University, Amman, Jordan for the financial support granted to cover the publication fees of this research article.

\section{REFERENCES}

[1] M Wu, Wenlan, Xianbin Wen, Haixia Xu, Liming Yuan, and Qingxia Meng. "Efficient range-free localization using elliptical distance correction in heterogeneous wireless sensor networks." International Journal of Distributed Sensor Networks 14, no. 1 (2018): 1550147718756274.

[2] Sohraby, Kazem, Daniel Minoli, and Taieb Znati. Wireless sensor networks: technology, protocols, and applications. John Wiley \& Sons, 2007.

[3] Akyildiz, I.F., \& Vuran, M.C., (2002), Wireless sensor networks, $1^{\text {st }}$ ed, UK: John Wiley \& Sons. 
[4] Abusaimeh, H., \& Yang, S. H. (2012). Energy-aware optimization of the number of clusters and cluster-heads in WSN. In Innovations in Information Technology (IIT), 2012 International Conference on (pp. 178-183). IEEE.

[5] Abusaimeh, h., (2014), "Balancing the Network Clusters for the Lifetime Enhancement in Dense Wireless Sensor Networks" in Arabian Journal for Science and Engineering, 2014. DOI 10.1007/s13369-0141059-x.

[6] chen, y., \& yango, c., (2006), A RSSI-Based Algorithm for Indoor Localization Using ZigBee in Wireless Sensor Network, Int. J. Digit. Content Technol. it's Appl. Digit. Content Technol. its Appl., 5(7), 407416.

[7] Larranaga, J.,\& Muguira, L.,\& jyyh \& Lopez-Garde, J. M. \& Vazquez, J. I. ,(2010), An Environment Adaptive ZigBee-Based Indoor Positioning Algorithm, 2010 Int. Conf. Indoor Position, Indoor Navi.
[8] Chu, C. H., \& Wang, Liang, C. H. C. K. W. \& Ouyang, J. H. \& Cai, \& Chen, Y. H., (2011), High-accuracy indoor personnel tracking system with a ZigBee wireless sensor network, 7th Int. Conf. Mob. Ad-hoc Sens. Networks, MSN 2011.

[9] Chandane, M. M., (2012), Performance Analysis of IEEE 802 . 15 . 4, Int. J. Comput. Appl., 40(5), 23-29.

[10] Obaid, T.,\& Abou-Elnour, A., \& Rehan, M., \& Muhammad Saleh, M. \& Tarique, M., (2014), Zigbee Technology and Its Application in Wireless Home Automation Systems: a Survey, Int. J. Comput. Networks Commun, 6(4), 115-131.

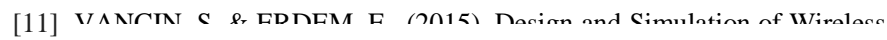
Sensor Network Topologies Using the ZigBee Standard. International Journal of Computer Networks and Applications. 2(3), 125-143.

[12] Shinde, V. \& Panchal, R. \& Panchal, J., (2016), ZigBee based indoor location tracking system. International Journal of Advanced Research in Electrical, Electronics and Instrumentation Engineering. 5(4). 